\title{
Visualization of spatial electrochemical activity via a combined thermal-electric potentiostat
}

\begin{abstract}
Authors: Hugo-Pieter Iglesias van Montfort, ${ }^{1}$ Thomas Burdyny ${ }^{1 *}$
Affiliations: 1 Materials for Energy Conversion and Storage (MECS), Department of Chemical Engineering, Faculty of Applied Sciences, Delft University of Technology, $2629 \mathrm{HZ}$ Delft, the Netherlands

*Correspondence to: t.e.burdyny@tudelft.nl

Abstract: The electrolysis of water, $\mathrm{CO}_{2}$ and $\mathrm{N}_{2}$ provide options for producing fossil-free fuels and feedstocks at global scales. Technological advancements are challenged by the complexity of phenomena spanning broad physical scales (angstroms to meters) and scientific domains. Further, activity is presently quantified indirectly, hindering disambiguation of catalytic and system effects. Here, we present a spatial thermal-electric potentiostat (STEP) which links local electrochemical activity to an associated operando heat signature. The STEP then directly maps catalytic activity with fine resolutions in temperature $(10 \mathrm{mK})$, time $(0.2 \mathrm{~s})$ and space $(0.1 \mathrm{~mm})$, capturing operational phenomena as they occur. We demonstrate STEP's potential for catalyst screening, degradation measurements and spatial mapping through water and $\mathrm{CO}_{2}$ electrolysis experiments up to $0.2 \mathrm{~A} \mathrm{~cm}^{-2}$. We identify rapid catalytic temperature spikes with activity $\left(>10 \mathrm{~K}^{2}\right.$ at $0.2 \mathrm{~A} \mathrm{~cm}^{-2}$ ) and localized activity fluctuations in operation, both which challenge many perceptions of the electrocatalyst and reaction environment during operation.
\end{abstract}

One Sentence Summary: An infrared imaging technique demonstrates a useful relationship between temperature change and electrochemical reaction rate 
Main Text: In the critical drive to find solutions for sustainable energy storage, electrochemical technologies which can be operated at global energy scales using fossil-free electricity offer promise. Research activities range broadly from the development of catalysts for novel reactions in nitrogen-based electrochemistry, steady improvement in $\mathrm{C}_{2}$ and $\mathrm{C}_{3}$ product selectivity for $\mathrm{CO}_{2}$ reduction (1), to the fine-tuning of well-understood reactions such as water-splitting.(1-4) Performance metrics such as current density, efficiency/overpotential, selectivity and stability provide the central foundation for evaluating electrochemical advancements and comparing systems.(5-7)

Despite catalytic activity being a spatial and temporal property (4D in space and time), the foundational metrics used as performance markers are measured as black box averaged quantities by potentiostats and bulk product quantification methods - distilling all spatial information to 1D resolutions in time. Not only are spatial resolutions in activity and selectivity lost, which results in phenomena and system behavior being indirectly evaluated, but electrochemistry is then faced with a one potentiostat - one data point problem. Catalyst screening efforts and mass data production for machine learning algorithms then subsequently suffer from insufficient or oversimplified data. Further, the dominant factors contributing to observed performance must be determined through multiple experiments and post-electrolysis analysis to properly disambiguate overlapping contributions of the catalyst, system and operating parameters. With electrochemical behavior governed by phenomena spanning broad physical scales (angstroms to meters) and scientific domains, a more direct link between electrochemical activity - and how we measure that activity - is necessary.

Efforts towards the measurement of temporal-spatial electrochemical activity, as well as combinatorial setups, have been introduced to partially address these shortfalls, with an emphasis on catalyst activity.(8-15) However, most approaches still require the sequential testing of miniaturized reactors or cell segmentation, both of which have spatial resolutions set by physical limitations.(16-19) Multi-well dye-based techniques allow for parallelization but provide only indirect indicators of electrochemical activity over a small catalyst, with tested current densities up to $-50 \mathrm{~mA} \mathrm{~cm}^{-2}$. (20) Dye techniques further rely on an observable liquid electrolyte resulting in cell configurations distanced from standard operation. Separately, thermographic approaches have been demonstrated in 'quasi in-situ' operation for identifying membrane defects and hydrogen crossover.(21-24) If an operando and accessible technique provided optical-level resolution of electrochemical activity under representative conditions, it would not only be valuable for catalyst testing, but broadly beneficial for both fundamental and applied analyses of the many rapidly advancing electrochemical fields. In this study we present a spatial thermal-electric potentiostat 
(STEP) which observes location-specific catalytic activity by exploiting the typically undesired energy inefficiencies inherent in electrochemical reactions.

\section{Heat as a proxy for electrochemical activity}

In considering a means of directly probing localized reactions, we reflected that all electrochemical reactions are a result of charge transport. Subsequently the current density (j) measured in our external circuits is the cumulative sum of all localized charge transport over an electrode's surface, and the voltage (V) represents the overpotentials needed to drive this transport. By nature, however, transport itself is fundamentally inefficient; a by-product of inefficient transport is heat generation. Importantly the quantity of heat produced (q) at a catalyst is linearly proportional to the amount of charges which are transferred in the electrochemical reaction $(q \propto j)$.(25) Thus, for an individual nanoparticle or region of an electrode, the local activity that is occurring should result in a proportional local heat generation. A characterization system capable of observing local heating can then in principle act as a proxy for electrochemical activity itself, opening the door for spatial and temporal mapping of catalytic activity with optical resolutions.

Such postulations are challenged by two facts: (i) heat is not an intrinsically measurable quantity, and (ii) associating heat with electrochemical activity requires disambiguating all heat sources present in electrochemical systems. Heating occurs across a device due to charge transport resistances in the electrode, electrolyte and membrane for example. An operando analysis technique is then required to demonstrate the theoretical link between heat and activity under actual operating conditions. Providing such a link would provide real-time visualized activity of an electrocatalyst, opening a door to new insights and experimental techniques.

We began by analyzing standard electrochemical characterization data to determine the validity and versatility of using heat generation of the electrocatalyst as a measure of electrochemical reaction rates. Specifically, the approach must meet the following stringent criteria: (i) heat production must be sufficient to observe activity at low potentials/current densities, (ii) heat generation must be translatable to a measurable intrinsic property (e.g., temperature), (iii) the location of temperature measurement is representative of the electrocatalyst, and (iv) measured temperature changes can be ascribed to various sources of heat generation. To assess these criteria, we can take a representative reaction and system as a base case. Specifically, hydrogen evolution reaction (HER) in a commonly utilized three-compartment electrochemical flow-cell (Fig. $1, A$ and $B$ ). A linear voltammetry scan (Fig. 1C) of a platinum (Pt) electrocatalyst deposited onto a carbon gas-diffusion layer (GDL) provides a basis for calculation. 
Using known relations for heat generation (Eq. S1 and S2), Fig. 1C can be transformed to a curve showing the total cathode input power and heat generation as a function of current density (Fig. S1). At a current density of $-10 \mathrm{~mA} \mathrm{~cm}^{-2}, \sim 5 \mathrm{~mW} \mathrm{~cm}^{-2}$ of heat is then generated by the chargetransfer of the HER reaction (illustrated in Fig. 1D). At this current density the collective thermal mass of the Pt catalyst and carbon GDL support would have a heating rate of $\sim 0.5 \mathrm{~K} \mathrm{~s}^{-1}$ in the absence of heat transfer effects (see SI for calculations). These heating rates are easily measured using thermocouples or infrared cameras, despite the chosen low current density and efficient reaction (HER on $\mathrm{Pt}$ ) as a base case; our criteria (i) and (ii) from above are then satisfied.

Meeting criteria (iii) adds the complexity of determining where temperature can be measured while remaining representative of the catalyst. Here the back of the carbon GDL is the optimal choice as it allows the electrochemical cell to operate unimpeded, overcoming a disadvantage of other operando techniques. However, the GDL temperature will differ from the catalyst layer due to heat transfer. Specifically, dissipation can occur either through the back of the GDL, or through the electrolyte (Fig. 1B and Fig. S5). If the electrolyte removes a disproportionate amount of the generated heat, the GDL temperature becomes less representative of the catalytic activity. To compare the two heat transfer mechanisms, an analysis was performed using Fourier's Law and a thermal equivalent circuit model. The thermal conductivities and approximate conduction lengths of the GDL and electrolyte thermal pathways control the relative thermal flux away from the catalyst layer (see SI for details). We find that for an equivalent temperature difference, the GDL conduct 30-fold higher heat flux than the electrolyte thermal pathway. At steady state, the temperature at the back of the GDL would then be higher than the electrolyte, and similar to that of the catalyst, meeting criteria (iii). The described SI equations illustrate how the ratio of thermal currents varies with choice of flow conditions and catalyst support.

Lastly, we then turned our attention to criteria (iv) involving dissociating heat sources. Ideally only reaction-driven heat from the catalyst influences the catalyst/GDL temperature, but in operation heating will also come from ohmic losses (Fig. 1E), membrane transport and other chemical phenomena. Similar to the description for catalyst heating, these other heat sources again are a result of the inefficient transport of charged species in the system. Returning to the above $\mathrm{Pt}$ example, we find that only ohmic heating is expected to be substantial as a secondary heat source on the cathode side of the reactor (see SI). For the $1 \mathrm{M} \mathrm{KOH}$ catholyte and $15 \mathrm{~mm}$ thick catholyte channel, ohmic heating is predicted and plotted as a function of current density (Fig. S3). In Fig. 1F we compare the catalyst and ohmic heating, highlighting that the catalyst heating is dominant (>60\% of heat) over the entire $0-200 \mathrm{~mA} \mathrm{~cm}^{-2}$ range, and more dominant at lower reaction rates. When the catholyte channel is reduced to $1 \mathrm{~mm}$, catalyst heating in the cathode half of the reactor 
accounts for $>95 \%$ of heat up to $-200 \mathrm{~mA} \mathrm{~cm}^{-2}$. We then took criteria (iv) to be met, while also understanding that heat generation can be further disambiguated with stringent modelling and system analysis as needed.

\section{Spatial thermal-electric potentiostat proof-of-concept}

With all four criteria for linking heat to activity met even for an efficient reaction, we subsequently sought to design and implement a system capable of spatially measuring activity via temperature changes. For optical level spatial resolutions over larger areas, the temperatures at the back of a high emissivity GDL were recorded using an infrared camera (Fig. 2A, Fig. S13). Importantly, the operation and configuration of the original electrochemical cell remains unaltered through the use of a gas-tight IR-transparent window positioned in the gas channel, providing data representative of standard high performance metrics works in applications such as $\mathrm{CO}_{2}$ electrolysis. $(1,26)$

Coupled together, the cell, infrared camera, and potentiostatic data form the Spatial ThermalElectric Potentiostat (STEP). Shown in Fig. 2B we measured the GDL temperature of a sputtered Pt electrode in $1 \mathrm{M} \mathrm{KOH}$ during a current density ramp rate of $-0.45 \mathrm{~mA} \mathrm{~cm}^{-2} \mathrm{~s}^{-1}$. Over a current density range of 0 to $-40 \mathrm{~mA} \mathrm{~cm}^{-2}$, relatively large temperature changes of $\sim 1.5 \mathrm{~K}$ are observed compared to the camera's resolution $(0.01 \mathrm{~K})$. Further, chronopotentiometry tests performed with the Pt electrode at -20 and $-200 \mathrm{~mA} \mathrm{~cm}^{-2}$ with elevated catholyte flow rates show a rapid GDL temperature change within $10 \mathrm{~s}$ of operation (Figs. S10 and S11). The catholyte and anolyte temperatures, however, only gradually increase despite the high flow rates. Importantly, upon the removal of an applied potential, the GDL temperature quickly decays back to that of the electrolyte temperature (Fig. S15). The rapid decrease highlights STEP's ability to measure both increasing and decreasing activity fluctuations during operation, while confirming that even though heat removal by the electrolyte is substantial, heat generation is even greater. We then confirm experimentally our criteria from above that measurable temperature changes are possible at low reaction rates, and GDL heat conduction is dominant versus the electrolyte. These results act as a proof-of-concept for the STEP system and highlight the fast response times possible with the developed technique.

We then turned to a $100 \mathrm{~nm}$ thick Ag sputtered catalyst deposited onto a GDL (see SI for details) to demonstrate the spatial-temporal capabilities of the STEP system under conditions known to cause failure via flooding of the GDL.(27) Here $\mathrm{N}_{2}$ gas is passed through the gas channel at the back of the GDL, and hydrogen evolution via water electrolysis takes place on the Ag catalyst. In Fig. 2C the current density is ramped from 0 to $-200 \mathrm{~mA} \mathrm{~cm}^{-2}$ at a rate of $-0.45 \mathrm{~mA} \mathrm{~cm}^{-2} \mathrm{~s}^{-1}$. Thermographic stills corresponding to timestamps of $-10,-20,-50,-100$ and $-200 \mathrm{~mA} \cdot \mathrm{cm}^{-2}$ highlight 
the rapid change in temperature as reaction rate increases, as well as the spatial effects occurring across the electrode. Near the end of the experiment, for example, perspiration of the electrolyte is observed. The lower temperatures of these droplets can be explained by evaporation of the water droplets by the non-humidified $\mathrm{N}_{2}$ stream. We note that the actual droplet temperatures are overestimated from those presented in Fig. $2 \mathrm{C}$, however, due to the different emissivity of water (0.98) versus the calibrated carbon emissivity $(0.81)$.

A critical takeaway from the Ag linear sweep, however, is that by the end of the $<8$ min experiment the temperature of the non-wetted portions of the GDL had already increased by $10 \mathrm{~K}$. Such a large temperature change would influence the ongoing electrochemical reaction kinetics. For example, kinetic studies on a Pt electrode in $0.1 \mathrm{M} \mathrm{KOH}$ for example showed an exchange current density change of almost 2-fold for temperature changes from just $298 \mathrm{~K}$ to $308 \mathrm{~K}$.(20) In electrochemical systems with competing reactions, large temperature changes would then also impact the relative reaction rates, influencing Faradaic efficiencies. For fields such as $\mathrm{CO}_{2}$ electrocatalysis, where current densities of $>1 \mathrm{~A} \mathrm{~cm}^{-2}$ are reported for single and multi-carbon products, the STEP system indicates that $10-30 \mathrm{~K}$ catalyst temperature swings are not unfathomable. These demonstrations of the STEP system highlight just how quickly and by how much electrocatalyst temperatures are elevated during operation, which is critical for mass transport, thermodynamic and kinetic models where temperatures are traditionally assumed as fixed quantities.

With the concept and response of the STEP system proven, we now provide a series of applications to both demonstrate the capabilities of the technique for comparing catalysts, spatial activity mapping and for different electrochemical reactions.

\section{Catalyst screening via overpotential-dependent temperature changes}

Thus far the STEP technique has focused on the link between heat and reaction rates. If our method can sense activity at the catalyst interface, the overpotential of the reaction should also be discernible through temperature measurements. Specifically, we asked if the technique can be a useful means of measuring both the onset potential of a given catalyst and comparing the activities of different materials when coupling the thermal data from the IR camera and electric data from the potentiostat.

Using the previously described Pt and Ag catalyst layers on GDL's, we observed temperature changes with the STEP system under increasing current densities of $-1 \mathrm{~mA} \mathrm{~s}^{-1}$ (Fig. S6). As an established catalyst for hydrogen evolution, Pt should generate more heat than the poor HER Ag 
catalyst at a fixed electrode potential, since the reaction rate would be higher. These assumptions are confirmed by comparing the thermal signal against the electrode potential, where at a fixed potential of $-0.8 \mathrm{~V}$ vs RHE the Pt catalyst has increased in temperature by $1 \mathrm{~K}$, while the relatively inactive Ag catalyst shows minimal increases (Fig. 3, B and C). The combination of thermal-electric data further shows the vast difference in onset potential of the two catalysts, demonstrating how STEP could be used to assess $\Delta T$ vs overpotential for numerous catalysts on the same electrode, an option not available with a potentiostat alone. The reversed scenario where temperature evolution is instead compared at fixed reaction rates has the opposite effect (Fig. 3D). Here the more efficient $\mathrm{Pt}$ catalyst shows a lower temperature change than $\mathrm{Ag}$ at a comparable reaction rate (Fig. 3, E and F).

The catalyst comparisons between $\mathrm{Ag}$ and $\mathrm{Pt}$ in Fig. 3 demonstrate the potential for screening catalysts in a combinatorial fashion on a singular GDL, using local temperature as a proxy for either reaction rate (Fig. 3B) or catalytic efficiency (Fig. 3E). Through designed experiments at each catalyst's location an effective temperature vs. reaction rate or overpotential can be plotted to identify the best catalyst, providing an accessible technique to avoid the one potentiostat - one data point challenge for single product reactions.

\section{Applications of spatial activity mapping}

Beyond catalytic screening applications, spatial mapping of electrolyzers provides an additional means of examining key transport phenomena, limiting chemical reactions, and changes in behavior over time. Uneven current distributions will result from poor catalyst deposition, differently ageing portions of the electrode, and spatially varying operating conditions (reactant concentrations, pressure, etc.), all of which are undesired. Defining a non-invasive probing mechanism to assess activity distribution is thus attractive, for both laboratory and scale-up efforts. Here we identify applications of the STEP technique for spatial mapping, as well as fundamental resolution limitations of the approach.

To simulate a catalyst region which may have been removed or deactivated during operation, we partially masked a portion of a GDL's microporous layer (MPL) prior to depositing a $100 \mathrm{~nm}$ thick copper $(\mathrm{Cu})$ catalyst (Fig. 4A). After a linear current density ramp to $-50 \mathrm{~mA} \mathrm{~cm}^{-2}$, a static current density was applied. Here distinctive heating patterns corresponding to the catalyst layer formed as a result of the electrochemical reactions occurring (Fig. 4B). The upper and lower hotter bands correspond to the coated sections of the GDE, while the cooler central region is the bare carbon MPL which has low activity. It can then be deduced that the static $-50 \mathrm{~mA} \mathrm{~cm}^{-2}$ applied to the 
system (112.5 mA total) is not equally distributed over the electrode, with the $\mathrm{Cu}$ regions experiencing much higher reaction rates.

The probing technique further allows for individual analysis per pixel, which means an image can be binned based on the individual value of each dot and tracked over time. Binning the readings results in a clearer picture (Fig. 4C), where we can see the central part of the GDE reading considerably lower temperatures than the other two bins. In this experiment the temperature distribution effects linked to the electrolyte flow patterns in the system can also be observed. Higher overall temperatures are binned at the exit side of the reactor (top-right), and in the stagnant electrolyte regions (bottom-right and top-left).

Following from the observations of the defected catalyst layer, we wanted to better understand how the catholyte and anolyte can influence the reaction rate distribution across a catalyst layer. To this end we varied the flow direction of the anolyte and catholyte flow from a bottom-to-top direction to a side-to-side direction. While in the cathode chamber product gases diffuse into the gas channel prior to nucleating, the anodic reaction performed here is the oxygen evolution reaction (OER) which results in substantial generation of $\mathrm{O}_{2}$ bubbles. In the side-to-side configuration gas bubbles become trapped in the system, resulting in a noticeably less stable linear sweep voltammetry measurement (Fig. 4D). Such disturbances in the anode compartment are shown to cause rapidly changing temperature profiles of the cathode GDL, implying that the shielding of $\mathrm{O}_{2}$ bubbles impacts the current density distribution of the cathode. During operation cloud-like heating patterns are observed to move across the cathode even though no bubbles are present in the catholyte (Fig. 4E and Fig. S14), which is not observed during bottom-to-top flow. These variations are best viewed in supplementary videos (see SI videos 1 and 2) which show that in both configurations fluid flow and gas evolution result in substantial variability at fixed locations. Such increases and decreases in activity would not only result in faster catalyst ageing, but also indicate that voltage and current density of an electrocatalyst is highly variable.

The collective spatial observations presented in this section point to many experimental systems having a less homogenous reaction environment than indicated through purely potentiostatic data. These considerations are particularly important within elevated current density experiments where large reaction rate changes occur with minimal changes in overpotential, implying a higher variance across the electrode's surface. The STEP technique is then valuable for assessing the homogeneity of systems and highlighting changes in activity as conditions are altered. 


\section{Exothermic homogeneous reactions during $\mathrm{CO}_{2}$ electrolysis}

Thus far we have highlighted STEP's potential to probe both electrocatalysts and systems with new perspectives, using hydrogen evolution as a demonstrative reaction. Here we highlight STEP's potential in other applications, namely $\mathrm{CO}_{2}$ electrolysis.

A known unwanted side-reaction in $\mathrm{CO}_{2}$ electrolyzers is the reaction of reactant $\mathrm{CO}_{2}$ with byproduct hydroxide, which lowers device utilization and represents one of the technology's largest practical barriers. $(7,28,29)$ The parasitic reaction which forms carbonates and precipitates is highly exothermic in nature and occur within the catalyst layer (Fig. 5A). The generated heat then should be discernible with STEP. Using a Ag catalyst that is adept at $\mathrm{CO}_{2}$ conversion to $\mathrm{CO}$, we compared the thermal-electric data of the catalyst in both a $\mathrm{CO}_{2}$ and $\mathrm{N}_{2}$ environment. $\mathrm{A} \mathrm{CO}_{2}$ feed will produce primarily $\mathrm{CO}$, while an $\mathrm{N}_{2}$ feed only produced $\mathrm{H}_{2}$. First observing the electrical data, a lower overpotential is shown for the $\mathrm{CO} 2$ gas-flow, which can be explained by Ag being a better CO2RR then HER catalyst (Fig. 5B). When observing the thermal data (Fig. 5C), however, 1-2 K greater temperatures are observed in the CO2RR case. These temperature changes are a result of the interaction between $\mathrm{CO}_{2}$ and hydroxide and occur despite the $\mathrm{CO}_{2}$ electrolysis having a more cathodic equilibrium potential (-0.11 $\mathrm{V}$ vs $\mathrm{RHE})$.

Another interesting observation that can be made from the electrode's average temperature (Fig. 5B), is the fact that the temperature rapidly increased to values $\sim 10 \mathrm{~K}$ above room temperature. Much like in the HER case above, these surface temperature variations have implications for mass transport and density-functional theory models which presently do not consider activity-temperature relationships.(30-32) For example the solubility of $\mathrm{CO}_{2}$ in water decreases by $\sim 30 \%$ from $298 \mathrm{~K}$ to $308 \mathrm{~K}$, while solubility limits for precipitation salt will increase.

For complex catalytic pathways observed in $\mathrm{CO}_{2}$ reduction, heat effects are also of noticeable influence on the selectivity of the catalyst. Copper electrodes have shown to shift towards ethylene production upon increasing operational temperature, but these reports do not consider local temperatures at the catalyst.(33,34) On top of this, improved mass transfer of reactants can be expected at these hot-spots, as viscosity of water and diffusion of gases in the interface are affected by temperature. $(35,36)$ For scale-up purposes, maintaining similarity of electrochemical activity across a surface is necessary to ensure understanding of the behavior of system. $(37,38)$ The ability of our system to indirectly scope activity over a surface for an electrolyser during operation presents a chance to gain more information from experiments, aiding in catalyst and system advancements. 


\section{Conclusion}

The concept of utilizing infrared emissions as a direct indicator of catalytic activity provides a broad set of potential applications for the ever-growing set of novel electrochemical reactions under investigation. The high emissivity of common carbon backbones provides an operando time and location specific measure of activity at optical resolutions, which can be coupled with electrical data analysis. Through a series of demonstrative applications, we show the developed STEP technique's ability to link measured changes in the gas-diffusion layer temperature to reaction overpotentials, catalyst type, defect sites on the catalyst layer, and dissolution of $\mathrm{CO}_{2}$ into the electrolyte during $\mathrm{CO}_{2}$ electrolysis. The substantial catalytic temperatures observed during regular operation highlight the need to reinterpret assumed kinetic data and reaction environments. The approach is shown to be both versatile, accessible and cost-effective.

\section{References:}

1. F. P. García de Arquer, C. T. Dinh, A. Ozden, J. Wicks, C. McCallum, A. R. Kirmani, D. H. Nam, C. Gabardo, A. Seifitokaldani, X. Wang, Y. C. Li, F. Li, J. Edwards, L. J. Richter, S. J. Thorpe, D. Sinton, E. H. Sargent, $\mathrm{CO} 2$ electrolysis to multicarbon products at activities greater than $1 \mathrm{~A}$ cm-2. Science (80-. ). (2020), doi:10.1126/science.aay4217.

2. T. Kim, G. T. R. Palmore, A scalable method for preparing Cu electrocatalysts that convert $\mathrm{CO} 2$ into C2+ products. Nat. Commun. 11 (2020), doi:10.1038/s41467-020-16998-9.

3. G. F. Chen, Y. Yuan, H. Jiang, S. Y. Ren, L. X. Ding, L. Ma, T. Wu, J. Lu, H. Wang, Electrochemical reduction of nitrate to ammonia via direct eight-electron transfer using a copper-molecular solid catalyst. Nat. Energy. 5, 605-613 (2020).

4. Z. Y. Wu, M. Karamad, X. Yong, Q. Huang, D. A. Cullen, P. Zhu, C. Xia, Q. Xiao, M. Shakouri, F. Y. Chen, J. Y. (Timothy) Kim, Y. Xia, K. Heck, Y. Hu, M. S. Wong, Q. Li, I. Gates, S. Siahrostami, H. Wang, Electrochemical ammonia synthesis via nitrate reduction on Fe single atom catalyst. Nat. Commun. 12 (2021), doi:10.1038/s41467-021-23115-x.

5. U. O. Nwabara, E. R. Cofell, S. Verma, E. Negro, P. J. A. Kenis, Durable Cathodes and Electrolyzers for the Efficient Aqueous Electrochemical Reduction of $\mathrm{CO} 2$. ChemSusChem (2020), , doi:10.1002/cssc.201902933.

6. K. Chan, A few basic concepts in electrochemical carbon dioxide reduction. Nat. Commun. 11 (2020), , doi:10.1038/s41467-020-19369-6. 
7. J. A. Rabinowitz, M. W. Kanan, The future of low-temperature carbon dioxide electrolysis depends on solving one basic problem. Nat. Commun. 11 (2020), , doi:10.1038/s41467-02019135-8.

8. E. Reddington, A. Sapienza, B. Gurau, R. Viswanathan, S. Sarangapani, E. S. Smotkin, T. E. Mallouk, Combinatorial Electrochemistry: A Highly Parallel, Optical Screening Method for Discovery of Better Electrocatalysts. J. Electron Spectrosc. Relat. Phenom. 178 (1986), p. 87.

9. F. G. Welsch, K. Stöwe, W. F. Maier, Fluorescence-based high throughput screening for noble metal-free and platinum-poor anode catalysts for the direct methanol fuel cell. ACS Comb. Sci. 13, 518-529 (2011).

10. J. L. Fernández, V. Raghuveer, A. Manthiram, A. J. Bard, Pd-Ti and Pd-Co-Au electrocatalysts as a replacement for platinum for oxygen reduction in proton exchange membrane fuel cells. $J$. Am. Chem. Soc. 127, 13100-13101 (2005).

11. J. B. Gerken, S. E. Shaner, R. C. Massé, N. J. Porubsky, S. S. Stahl, A survey of diverse earth abundant oxygen evolution electrocatalysts showing enhanced activity from $\mathrm{Ni}-\mathrm{Fe}$ oxides containing a third metal. Energy Environ. Sci. 7, 2376-2382 (2014).

12. H. Ye, H. S. Park, A. J. Bard, Screening of electrocatalysts for photoelectrochemical water oxidation on W-doped BiVO4 photocatalysts by scanning electrochemical microscopy. J. Phys. Chem. C. 115, 12464-12470 (2011).

13. D. Guevarra, A. Shinde, S. K. Suram, I. D. Sharp, F. M. Toma, J. A. Haber, J. M. Gregoire, Development of solar fuels photoanodes through combinatorial integration of $\mathrm{Ni}$-La-Co-Ce oxide catalysts on BiVO4. Energy Environ. Sci. 9, 565-580 (2016).

14. S. E. Fosdick, S. P. Berglund, C. B. Mullins, R. M. Crooks, Evaluating electrocatalysts for the hydrogen evolution reaction using bipolar electrode arrays: $\mathrm{Bi}$ - and trimetallic combinations of Co, $\mathrm{Fe}, \mathrm{Ni}, \mathrm{Mo}$, and W. ACS Catal. 4, 1332-1339 (2014).

15. D. K. Kim, W. F. Maier, Combinatorial discovery of new autoreduction catalysts for the $\mathrm{CO} 2$ reforming of methane. J. Catal. 238, 142-152 (2006).

16. P. C. Ghosh, T. Wüster, H. Dohle, N. Kimiaie, J. Mergel, D. Stolten, In situ approach for current distribution measurement in fuel cells. J. Power Sources (2006), doi:10.1016/j.jpowsour.2005.03.219.

17. T. V. Reshetenko, G. Bender, K. Bethune, R. Rocheleau, A segmented cell approach for 
studying the effects of serpentine flow field parameters on PEMFC current distribution. Electrochim. Acta (2013), doi:10.1016/j.electacta.2012.10.103.

18. L. Osmieri, S. Mauger, M. Ulsh, K. C. Neyerlin, G. Bender, Use of a segmented cell for the combinatorial development of platinum group metal-free electrodes for polymer electrolyte fuel cells. J. Power Sources (2020), doi:10.1016/j.jpowsour.2020.227829.

19. R. Lin, H. Sander, E. Gülzow, A. Friedrich, Investigation of Locally Resolved Current Density Distribution of Segmented PEM Fuel Cells to Detect Malfunctions. ECS Trans. 26, 229-236 (2010).

20. J. L. Hitt, Y. C. Li, S. Tao, Z. Yan, Y. Gao, S. J. L. Billinge, T. E. Mallouk, A high throughput optical method for studying compositional effects in electrocatalysts for $\mathrm{CO} 2$ reduction. Nat. Commun. 12, 1-10 (2021).

21. A. Phillips, M. Ulsh, K. C. Neyerlin, J. Porter, G. Bender, Impacts of electrode coating irregularities on polymer electrolyte membrane fuel cell lifetime using quasi in-situ infrared thermography and accelerated stress testing. Int. J. Hydrogen Energy. 43, 6390-6399 (2018).

22. M. Wang, S. Medina, J. Ochoa-Lozano, S. Mauger, S. Pylypenko, M. Ulsh, G. Bender, Visualization, understanding, and mitigation of process-induced-membrane irregularities in gas diffusion electrode-based polymer electrolyte membrane fuel cells. Int. J. Hydrogen Energy. 46, 14699-14712 (2021).

23. P. K. Das, A. Z. Weber, G. Bender, A. Manak, D. Bittinat, A. M. Herring, M. Ulsh, Rapid detection of defects in fuel-cell electrodes using infrared reactive-flow-through technique. J. Power Sources. 261, 401-411 (2014).

24. N. V Aieta, P. K. Das, A. Perdue, G. Bender, A. M. Herring, A. Z. Weber, M. J. Ulsh, Applying infrared thermography as a quality-control tool for the rapid detection of polymer-electrolytemembrane-fuel-cell catalyst-layer-thickness variations. J. Power Sources. 211, 4-11 (2012).

25. H. A. Catherino, Estimation of the heat generation rates in electrochemical cells. J. Power Sources (2013), doi:10.1016/j.jpowsour.2013.03.169.

26. C. T. Dinh, T. Burdyny, G. Kibria, A. Seifitokaldani, C. M. Gabardo, F. Pelayo García De Arquer, A. Kiani, J. P. Edwards, P. De Luna, O. S. Bushuyev, C. Zou, R. Quintero-Bermudez, Y. Pang, D. Sinton, E. H. Sargent, CO2 electroreduction to ethylene via hydroxide-mediated copper catalysis at an abrupt interface. Science (80-. ). (2018), doi:10.1126/science.aas9100. 
27. K. Yang, R. Kas, W. A. Smith, T. Burdyny, Role of the Carbon-Based Gas Diffusion Layer on Flooding in a Gas Diffusion Electrode Cell for Electrochemical CO2 Reduction. ACS Energy Lett. 6, 33-40 (2020).

28. J. E. Huang, F. Li, A. Ozden, A. Sedighian Rasouli, F. P. García de Arquer, S. Liu, S. Zhang, M. Luo, X. Wang, Y. Lum, Y. Xu, K. Bertens, R. K. Miao, C.-T. Dinh, D. Sinton, E. H. Sargent, CO 2 electrolysis to multicarbon products in strong acid. Science (80-. ). 372, 1074-1078 (2021).

29. A. Ozden, Y. Wang, F. Li, M. Luo, J. Sisler, A. Thevenon, A. Rosas-Hernández, T. Burdyny, Y. Lum, H. Yadegari, T. Agapie, J. C. Peters, E. H. Sargent, D. Sinton, Cascade CO2 electroreduction enables efficient carbonate-free production of ethylene. Joule. 5, 706-719 (2021).

30. D. Bohra, J. H. Chaudhry, T. Burdyny, E. A. Pidko, W. A. Smith, Modeling the electrical double layer to understand the reaction environment in a $\mathrm{CO} 2$ electrocatalytic system. Energy Environ. Sci. 12, 3380-3389 (2019).

31. N. T. Nesbitt, T. Burdyny, H. Simonson, D. Salvatore, D. Bohra, R. Kas, W. A. Smith, LiquidSolid Boundaries Dominate Activity of CO2Reduction on Gas-Diffusion Electrodes. ACS Catal. 10, 14093-14106 (2020).

32. R. Kas, A. G. Star, K. Yang, T. Van Cleve, K. C. Neyerlin, W. A. Smith, Along the Channel Gradients Impact on the Spatioactivity of Gas Diffusion Electrodes at High Conversions during CO2 Electroreduction. ACS Sustain. Chem. Eng. 9, 1286-1296 (2021).

33. S. T. Ahn, I. Abu-Baker, G. T. R. Palmore, Electroreduction of CO2 on polycrystalline copper: Effect of temperature on product selectivity. Catal. Today. 288, 24-29 (2017).

34. Y. Hori, K. Kikuchi, A. Murata, S. Suzuki, Production of methane and ethylene in electrochemical reduction of carbon dioxide at copper electrode in aqueous hydrogencarbonate solution. Chem. Lett., 897-898 (1986).

35. E. N. Fuller, P. D. Schettler, J. C. Giddings, A new method for prediction of binary gas-phase diffusion coefficients. Indsutrial Eng. Chem. 58, 18-27 (1966).

36. J. Kestin, M. Sokolov, W. A. Wakeham, Viscosity of liquid water in the range $-8^{\circ} \mathrm{C}$ to $150^{\circ} \mathrm{C} . \mathrm{J}$. Phys. Chem. Ref. Data. 7, 941-948 (1978).

37. G. Prentice, Electrochemical engineering principles (Prentice Hall, New Jersey, 1990). 
38. F. Goodridge, K. Scott, Electrochemical Process Engineering (Plenum Press, 1995). 


\section{Funding:}

HP.IvM. and T.B. would like to acknowledge the co-financing provided by Shell and PPP-allowance from Top Consortia for Knowledge and Innovation (TKI's) of the Ministry of Economic Affairs and Climate in the context of the e-Refinery Institute. T.B. would also like to acknowledge the NWO for an individual Veni grant.

\section{Author Contributions:}

HP.IvM. completed all the experiments and modelling work, TB and HP.IvM. conceived the project. All authors contributed to writing and editing the manuscript.

\section{Competing Interests:}

HP.IvM. and T.B. of the Delft University of Technology have filed provisional patent application no. 2028898 regarding the method and configuration for observing catalytic activity via infrared radiation.

\section{Acknowledgements:}

The authors would also like to acknowledge Joost Middelkoop for assistance with setting up the experimental systems for product detection and Herman Schreuders for mentioning we "had an old IR-camera' lying around and sparking the idea for this project.

Data and Materials Availability:

The data used in this work is available upon request.

\section{List of Supplementary Materials:}

Supplementary Materials

Materials and Methods

Fig $\mathrm{S} 1$ - S15

References (39 - 44)

Movie S1\& S2 
A

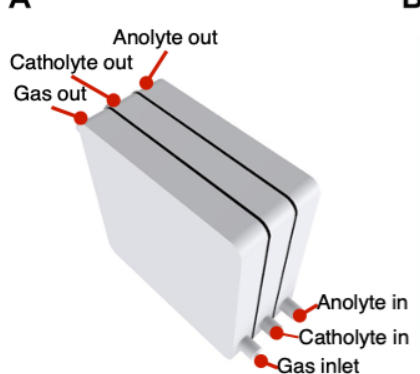

D

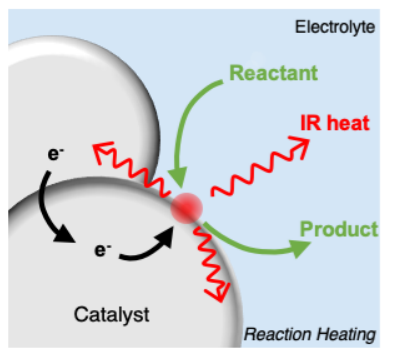

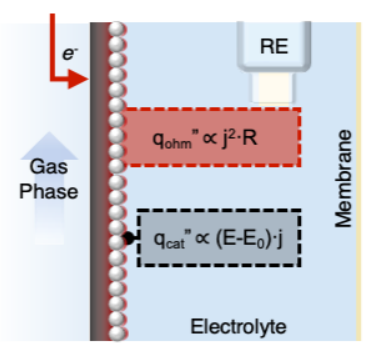

C

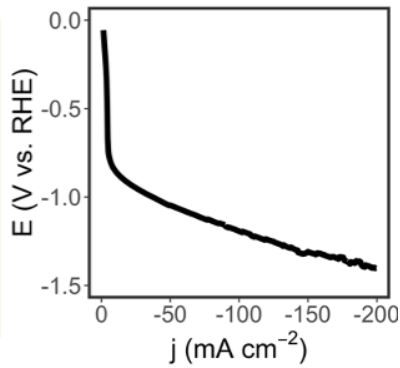

$\mathbf{F}$

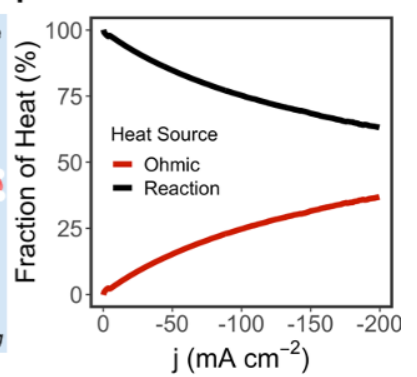

Fig. 1. Heat production in a lab-scale electrolyzer is controlled mainly by catalytic and resistive overpotentials. (A) Schematic representation of a representative 3-compartment electrolyzer cell. (B) Half-cell view of an electrochemical cell with a flowing catholyte showing the heat generation locations and formula of ohmic heating ( $q_{\mathrm{ohm}}$ ) and reaction-driven heating (qcat). (C) Polarization curve of a Pt GDE during HER in the reported electrolyzer. (D) Heat is produced at the catalyst-electrolyte interface due to the overpotential required to drive the reaction. (E) Ohmic heating as a result of ion transport in an electrolyte. (F) Relative source of heating at the interface as a function of applied current density for a $15 \mathrm{~mm}$ thick $1 \mathrm{M} \mathrm{KOH}$ electrolyte. 

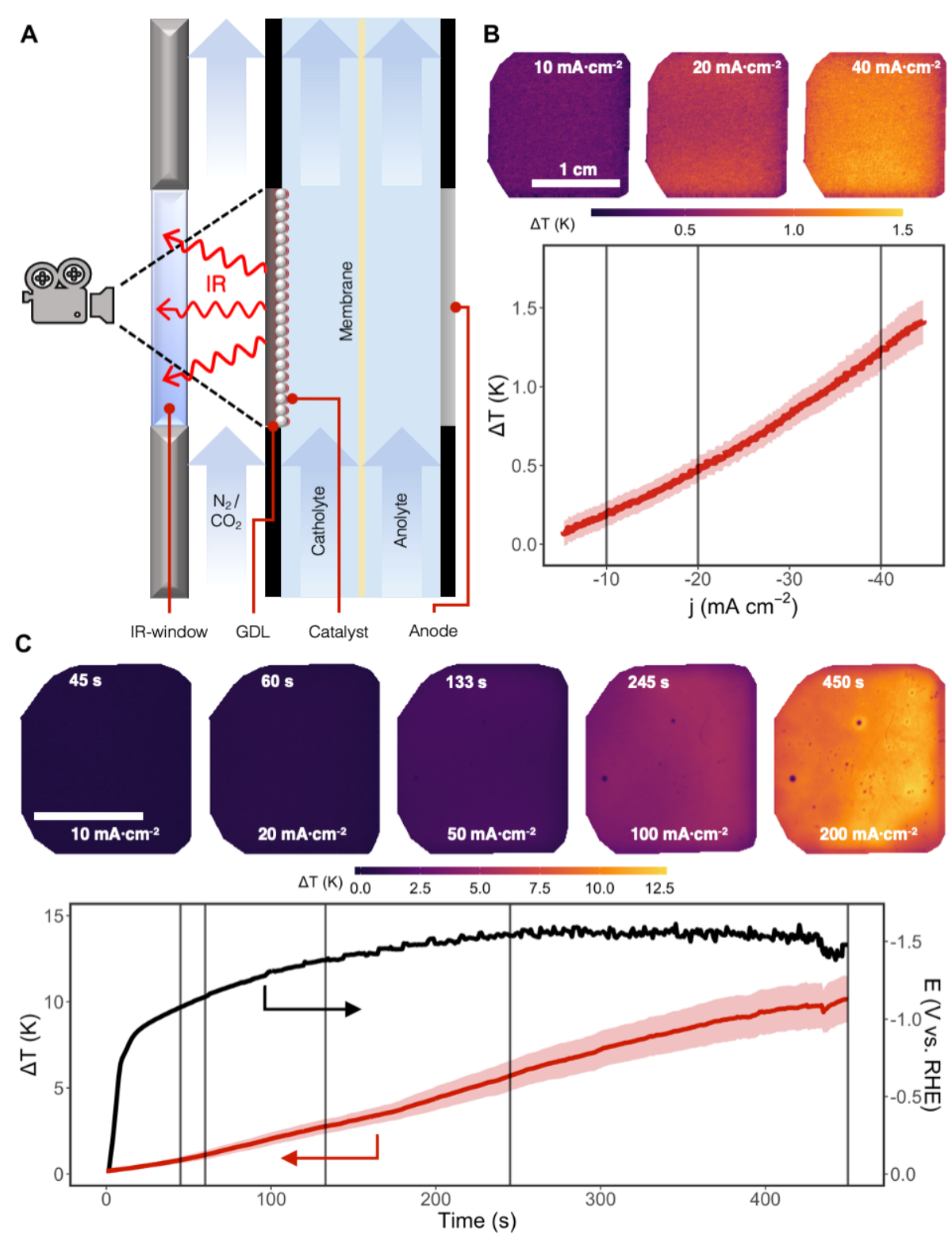

Fig. 2. A windowed electrolyzer design allows to sense the catalytic activity of a gas-diffusion electrode. (A) Schematic depiction of the windowed electrolyzer. (B) Thermographic stills of the cathode with a stagnant electrolyte layer at low current densities. $100 \mathrm{~nm}$ Pt in $1 \mathrm{M} \mathrm{KOH}$. (C) Average, maximum and minimum temperatures of the cathode backbone during polarization curve collection as a function of time. 100nm Ag catalyst in $1 \mathrm{M} \mathrm{KOH}$. Thermographic stills corresponding to the polarization curve, time of acquisition and corresponding current densities as indicated. 
A

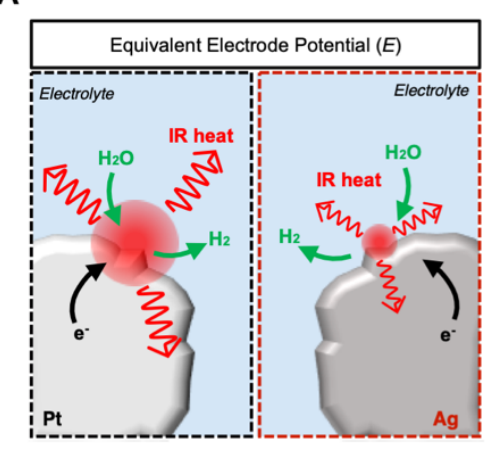

D

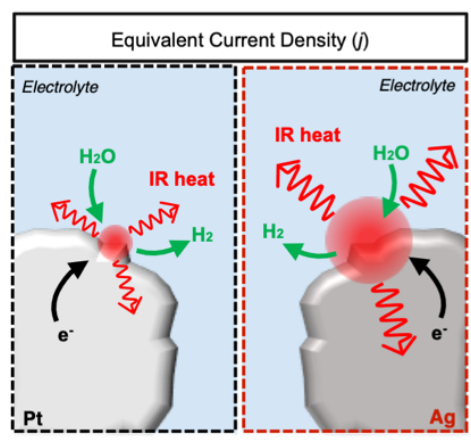

B

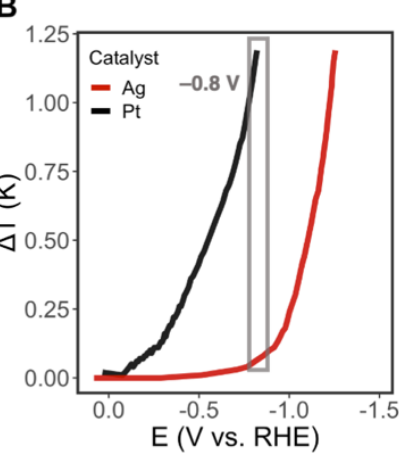

E

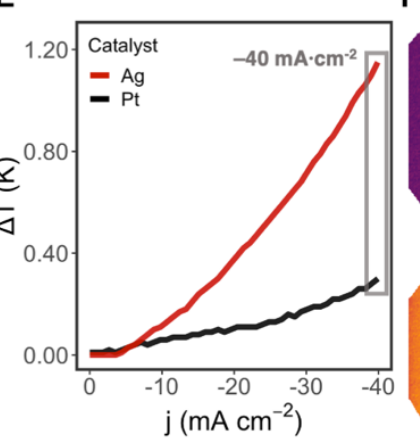

C

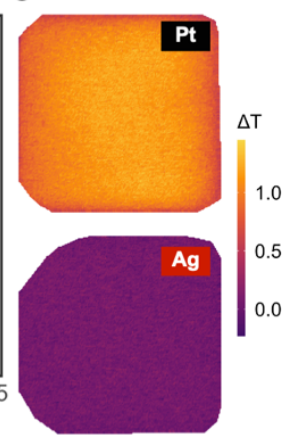

F

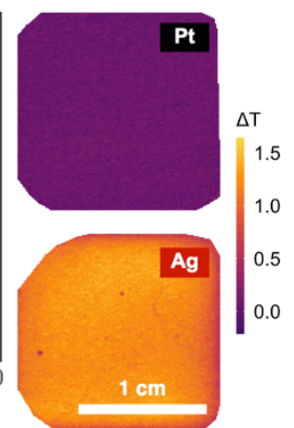

Fig. 3. Electrode activity can be visualized using infrared thermography. (A) At equal potential, the higher activity on platinum will cause a higher rate of heat generation. (B) Potential dependence of the average temperature observed on the $\mathrm{Pt}$ and $\mathrm{Ag}$ GDE's, with $1 \mathrm{M} \mathrm{KHCO}_{3}$ as the electrolyte flowing at $6 \mathrm{sccm}$. (C) Thermographic stills of the Pt and Ag GDE's at -0.8 V vs. RHE. (D) At equal current density, the lower activity of silver will cause a higher heating rate due to increased overpotential. (E) Current density dependence of the average temperature observed on the Pt and Ag GDE's using the same electrolyte. $(F)$ Thermographic stills of the Pt and Ag GDE's at $-40 \mathrm{~mA} \mathrm{~cm}^{-2}$. 
A

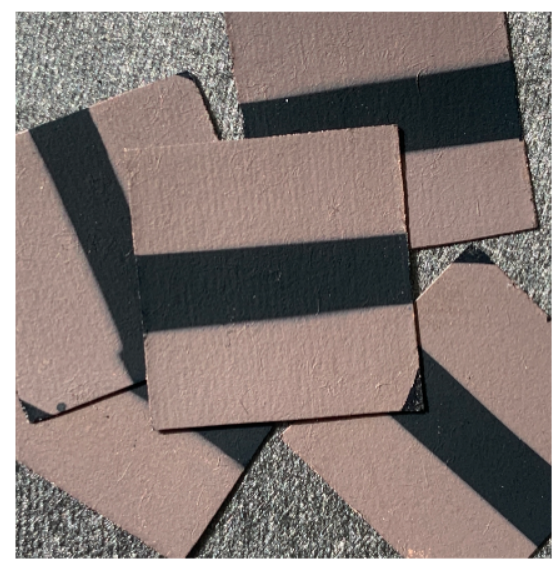

C

B

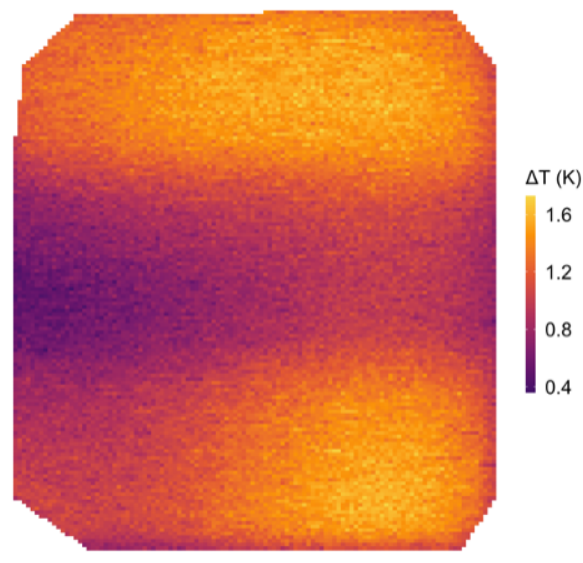

$\Delta \mathrm{T}<1.0 \mathrm{~K}$

$1.0 \leq \Delta T<1.3 \mathrm{~K}$

$\Delta \mathrm{T} \geq 1.3 \mathrm{~K}$

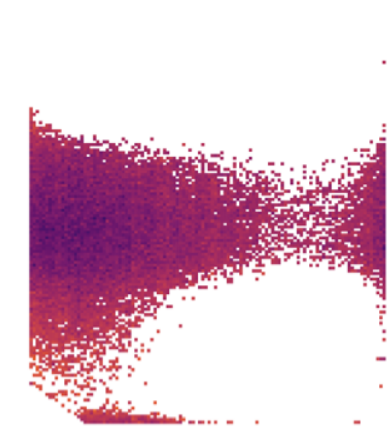

D

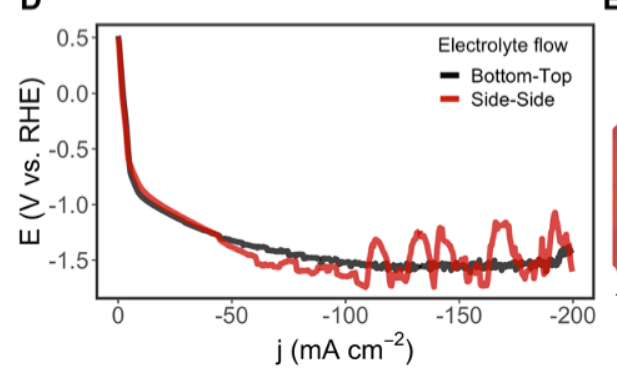

E
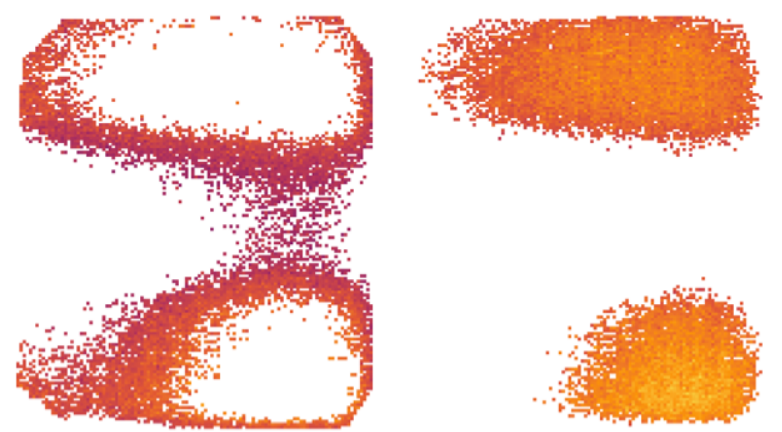

Bottom-Top

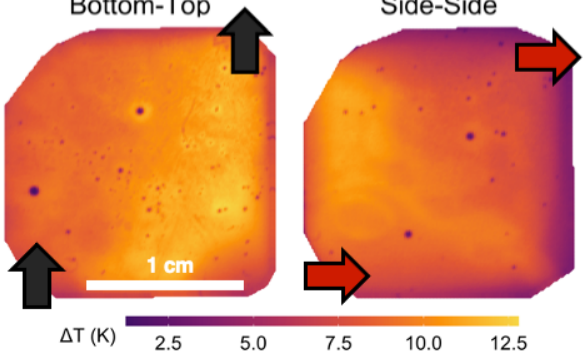

Fig. 4. IR thermography is effective for defect detection and sensing of current density distribution over the catalyst surface. (A) Gas-diffusion electrodes with defects applied by masking during deposition. (B) Thermographic still of a defected Cu-GDE at $50 \mathrm{~mA} \mathrm{~cm}^{-2}$ with $1 \mathrm{M} \mathrm{KOH}$ flowing at $6 \mathrm{sccm}$. (C) Binned individual pixels of the still image in $\mathbf{B}$ as a function of temperature increase. (D) Polarization curves under the same electrolyte flow directions in $\mathbf{E}$, where the influence of bubble accumulation can be observed in the form of noise. (E) Thermographic stills of a 100nm Ag electrode with different electrolyte flow configurations at $200 \mathrm{~mA} \mathrm{~cm}^{-2}$. 
A

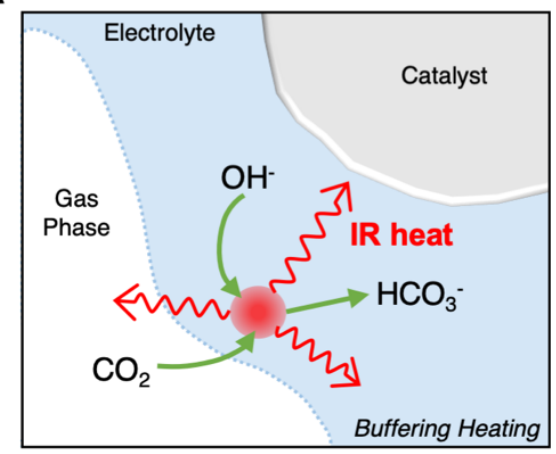

B

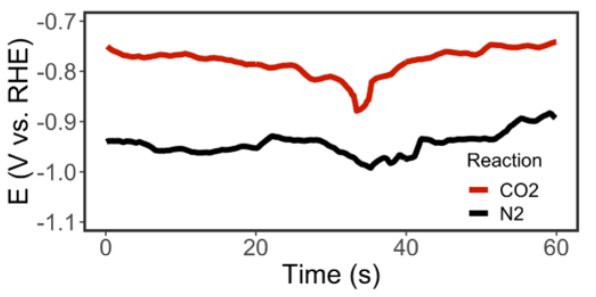

C

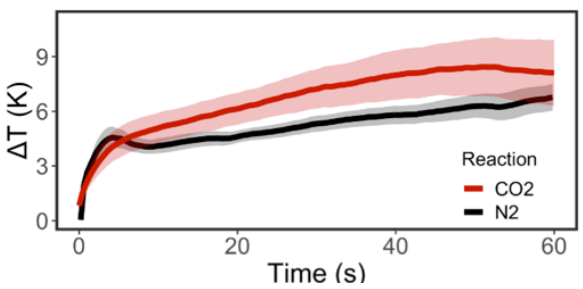

Fig. 5. The heat effects of $\mathrm{CO}_{2}$ dissolution are noticeable even at high current densities. (A) $\mathrm{CO}_{2}$ dissolves in alkaline media to form bicarbonate in an exothermic reaction. (B) Reaction potentials for HER and CO2RR at a 100nm Ag GDE using $1 \mathrm{M} \mathrm{KOH}$ at $200 \mathrm{~mA} \mathrm{~cm}^{-2}$. (C) Temperature increases over the GDE surface for the sequences in $\mathbf{B}$, where the CO2RR displays a noticeably higher overall temperature. 